\title{
The GEO 600 core optics
}

\author{
W. Winkler ${ }^{\text {a,* }}$, K. Danzmann ${ }^{\mathrm{a}, \mathrm{b}}$, H. Grote ${ }^{\mathrm{a}}$, M. Hewitson ${ }^{\mathrm{a}}$, S. Hild ${ }^{\mathrm{a}}$, J. Hough ${ }^{\mathrm{c}}$, \\ H. Lück ${ }^{\text {b }}$, M. Malec ${ }^{\text {b }}$, A. Freise ${ }^{\mathrm{d}}, \mathrm{K}_{\text {. Mossavi }}{ }^{\mathrm{a}}$, S. Rowan ${ }^{\mathrm{c}}$, A. Rüdiger ${ }^{\mathrm{a}}$, R. Schilling ${ }^{\mathrm{a}}$, \\ J.R. Smith ${ }^{\mathrm{a}}$, K.A. Strain ${ }^{\mathrm{c}}$, H. Ward ${ }^{\mathrm{c}}$, B. Willke ${ }^{\mathrm{b}}$ \\ ${ }^{a}$ Max-Planck-Institut für Gravitationsphysik (Albert Einstein Institut), Hannover, Germany \\ ${ }^{\mathrm{b}}$ Institut für Gravitationsphysik der Universität Hannover, Germany \\ ${ }^{\mathrm{c}}$ Institute for Gravitational Research, University of Glasgow, UK \\ ${ }^{\mathrm{d}}$ School of Physics and Astronomy, The University of Birmingham, UK
}

Received 8 September 2006; received in revised form 27 June 2007; accepted 20 July 2007

\begin{abstract}
The optical layout of the interferometric gravitational wave detector GEO600 is described in detail. Criteria for the choice of the geometry of this power- and signal recycled interferometer are presented, including the beam shape inside the interferometer and the surface figure of the optical components. Light power limits for the present setup are discussed. In addition, the demands for the mode cleaners and their performance are given.
\end{abstract}

(C) 2007 Elsevier B.V. All rights reserved.

Keywords: Laser-interferometric gravitational wave detectors; Folded optical cavities; Stability criteria; Interference quality

\section{Introduction}

The interferometric gravitational wave detector GEO 600 has been operational in its designed mode of dual recycling since end of 2003 [1] (see below). In spring 2005 the power recycling mirror was changed to allow higher power build up in the interferometer [2]. The light power in front of the power recycling mirror is now (spring 2007) $3.2 \mathrm{~W}$, whereas the internal circulating power is $2.7 \mathrm{~kW}$. This paper gives a comprehensive description of the optics of the interferometer as it has been used so far.

We start with an overview, and then describe the core optics in more detail.

\section{Optical layout of GEO 600}

Basically GEO 600 is a Michelson interferometer to measure $\delta \ell / \ell$, the strain in space, as introduced by gravita-

\footnotetext{
${ }^{*}$ Corresponding author. Tel.: +49 9189409726.

E-mail address: walter.winkler@rzg.mpg.de (W. Winkler).
}

tional waves. (The amplitude of gravitational waves is defined by $h=2 \delta \ell / \ell$.) Contrary to most other experiments of this kind [3-5], there are no Fabry-Perot resonators in the arms, which are used to increase the storage time of the light, or, in other words, to increase the light path in the interferometer arms. Instead, in GEO 600 the light path is doubled in each arm by folding the light beam, and in addition the technique of signal recycling is implemented, which allows to tune the interferometer to a variety of signals. The frequency for the peak sensitivity can be chosen by the microscopic position of the signal recycling mirror, and the bandwidth of the sensitivity curve by adjusting the transmissivity of the recycling mirror [6].

The complete optical layout of GEO 600 is shown in Fig. 1. The light source is a $12 \mathrm{~W} \mathrm{Nd:YAG} \mathrm{laser} \mathrm{with} \mathrm{a}$ wavelength of $1064 \mathrm{~nm}$. In order to reduce geometrical beam fluctuations sufficiently, the light is sent through two triangular mode-cleaners in series (see Section 7). Their round trip path-length was chosen to be $8 \mathrm{~m}$ for the first one, and $8.1 \mathrm{~m}$ for the second one. The finesses of 2700 and 1900 , respectively, were due to the coating process as 


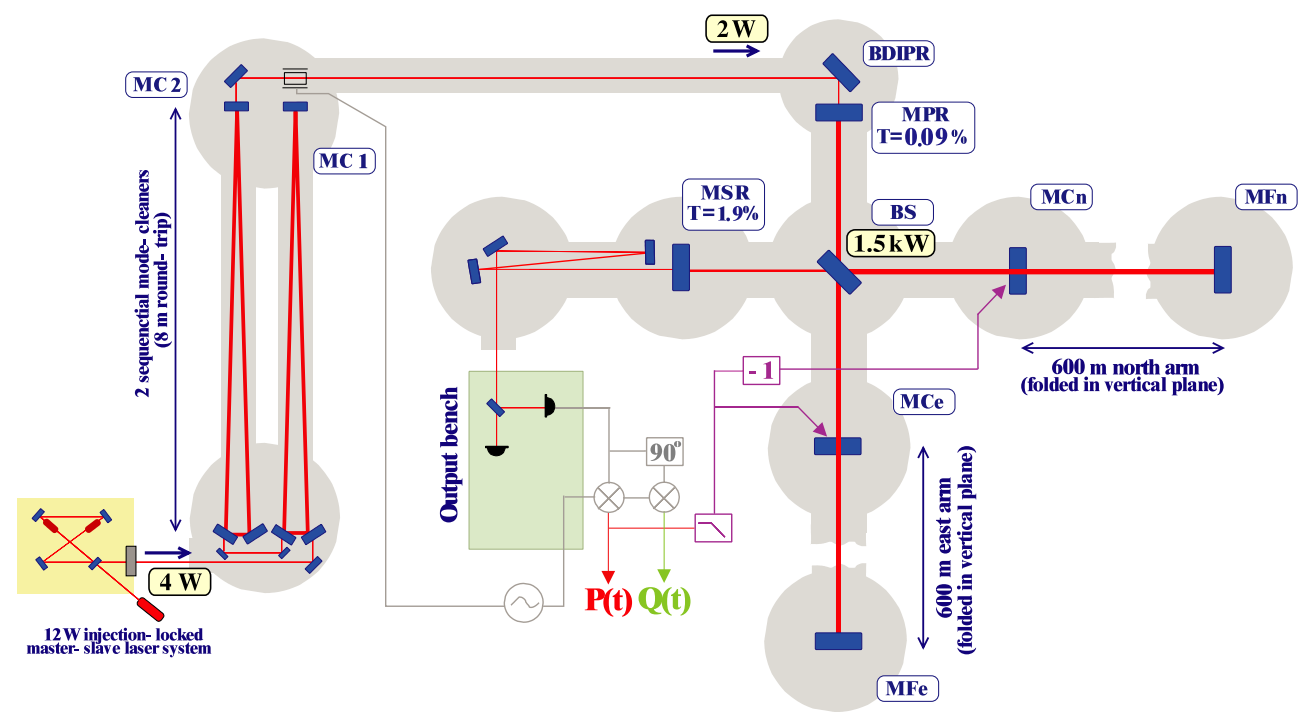

Fig. 1. Optical layout of GEO 600. Light from the laser bench, shown schematically, passes into the vacuum system (shaded area). The first main optical components form the two mode-cleaners, discussed in Sections 2 and 7 of the text. The beam emerging from the mode-cleaners is expected in a telescope, and directed into the "core optics" which are described in the text and shown in more detail in Fig. 2. The emergent light is detected on the output bench (shaded green) and converted to the two primary electrical output signals $P(t)$ and $Q(t)$ that are combined to form the signal channel in which the effects of gravitational waves should be seen. (For interpretation of the references in colour in this figure legend, the reader is referred to the web version of this article.)

applied by the manufacturer. The different lengths allowed us to sufficiently separate the eigenfrequencies from multiples of free spectral ranges of the cavities, which is the requirement for effective spatial filtering by the sequential mode-cleaners.

After passing through a suspended phase modulator and Faraday isolator the spatially filtered beam is injected into the interferometer through the power recycling mirror MPR (Suprasil 1, $150 \mathrm{~mm}$ diameter and $100 \mathrm{~mm}$ thick, see Fig. 2) and proceeds to the beam-splitter (260 mm diameter, $80 \mathrm{~mm}$ thick, Suprasil $311 \mathrm{SV}$ ). In each arm the beam is reflected at the far mirror and sent back to the near mirror, which is located in the central building $25 \mathrm{~cm}$ above the outgoing beam. (To show the light path more clearly, in Fig. 2 the near mirrors are drawn to sit beside the input beam.) The beam then retraces the light path back via the far mirror to the beam-splitter. Near and far mirrors are made from Suprasil 1, with a diameter of $180 \mathrm{~mm}$ and a thickness of $100 \mathrm{~mm}$. In principle a compensation plate would be desirable for symmetry reasons. But, in order to avoid the additional thermoelastic noise introduced by such a component, it was left out.

The main output beam consists of the light transmitted through the signal recycling mirror MSR.

The geometrical length of both arms is very close to $\ell=600 \mathrm{~m}$. Exact values were determined by measuring the resonance frequencies of the power- and signal recycling cavities (which had to be unfolded to obtain the separation between power recycling mirror and far mirror). This gave: Distance MPR - MFn $=599.71 \mathrm{~m}, \mathrm{MFn}-\mathrm{MCn}=597.01 \mathrm{~m}$; $\mathrm{MPR}-\mathrm{MFe}=599.73 \mathrm{~m}, \mathrm{MFe}-\mathrm{MCe}=597.07 \mathrm{~m}$. The phys-

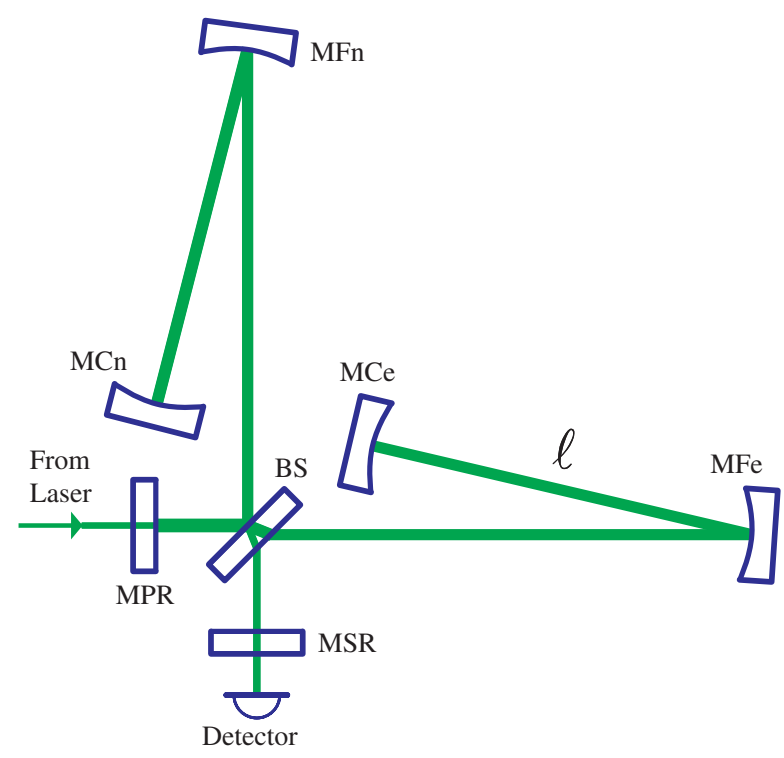

Fig. 2. Optical layout of the core optics in GEO 600. Apart from the central beam-splitter (BS) and the photo-detector (shown schematically), the remaining core components are mirrors (three letter name starting with "M"). For the mirrors forming the East and North arms the second letter of the name indicates whether the mirror is in the central station (C) or an end station $(\mathrm{F})$, while the final letter indicates whether the East (e) or North (n) arm is concerned. The power- and signal recycling mirrors are called MPR and MSR, respectively. The main properties of these mirrors are given in the text.

ical thickness of the beam-splitter is $80 \mathrm{~mm}$. Taking into account the angle of the beam and the refractive index of the fused silica material used, this gives an optical path of $13 \mathrm{~cm}$. 
Thus, the optical path-length is close to $2400 \mathrm{~m}$. The roundtrip path difference between the two arms is $13.7 \mathrm{~cm}$. This allows the readout of the interferometer at its dark-fringe output by phase modulation of the input light (Schnupp modulation).

In order to eliminate higher-order modes created inside the interferometer, the design of the vacuum system leaves room for an output mode-cleaner in the beam path, before the light goes to the photodiode. Higher-order modes originating from a mismatch of the interferometer do not carry a useful amount of gravitational wave information, but mostly contribute to the noise. The dominating contribution to these modes originates from an asymmetry in curvature of the mirror surfaces in the two arms. This effect was substantially reduced by thermal adjustment of the curvature of the far mirror in the east arm [7].

The design choices for the optical layout were

1. to include power- and signal recycling,

2. to keep the beam diameter near the input in the central tank small for several reasons - mostly to allow a separation of all relevant reflections at the beam-splitter and facilitate their use for other purposes,

3. to avoid resonances of higher-order modes of the laser light inside the recycling cavities by properly choosing the geometrical parameters defining the Gouy phase [8] (see also end of Section 6),

4. to make the interferometer insensitive to changes in orientation of the far mirrors, and finally

5. to allow for high light power.

Recycling techniques are realized by inserting a mirror in front of the interferometer and at its output. The power recycling cavity for instance is formed by the power recycling mirror MPR as one mirror of this cavity, whereas the other one is made up by the interferometer composed of beam-splitter together with the arm mirrors. The interferometer is kept at the dark-fringe operating point, and thus it acts for the carrier light as the second highly reflecting mirror of a two-mirror cavity, the power recycling cavity. The signal recycling cavity is formed analogously by the combination of the signal recycling mirror MSR and the interferometer; it enhances the signal sidebands containing the gravitational wave information. The practical implementations and the operation of those cavities, including auto-alignment and position control, have been described elsewhere [1]. Power- and signal recycling mirrors at present have a transmissivity of $0.09 \%$ and $1.9 \%$, respectively.

\section{The folded cavity}

The folded arm cavity may be represented by an equivalent linear cavity, $1200 \mathrm{~m}$ long, with an internal lens replacing the curved far mirror. The mirrors of this cavity are the power recycling (or signal recycling) mirror at one end, and the near arm mirror at the other. The properties of such a cavity have been described by Kogelnik in his original paper [9]. There one can find for instance the conditions to get a small beam diameter in the central tank (see Eq. (10) below), or the proper Gouy phases to avoid resonances of higher-order light modes inside the recycling cavities (see Eq. (24)).

In GEO 600 both arms are designed to be symmetrical (besides a tiny asymmetry in armlength to allow for Schnupp modulation). For our description let us take the east arm, for instance. MPR acts as coupling mirror, MCe as end mirror and $\mathrm{MFe}$ as the internal lens. The north arm is as symmetrical to the east arm as possible.

For a folded cavity similar relations hold as for the usual two-mirror cavity. The corresponding $G$-factors (written in capital letters for discrimination against the $g$-factors for two-mirror cavities [10]) are given by

$G_{1}=1-2 d_{2} / R_{1}-d_{0} / R_{0}$,

$G_{2}=1-2 d_{1} / R_{1}-d_{0} / R_{2}$.

$d_{1}$ and $d_{2}$ are the separation between MPR and MFe or $\mathrm{MFe}$ and MCe, respectively. In GEO 600 these distances are close to the armlength $\ell=600 \mathrm{~m}$, each. $d_{0}$ is defined through

$d_{0}=d_{1}+d_{2}-2 d_{1} d_{2} / R_{1}$.

The recycling mirrors are flat: $R_{0}=\infty$; the radius of curvature of the far mirrors MFe and MFn is designed to be $R_{1}=640 \mathrm{~m}$ and for the near mirrors $\mathrm{MCe}$ and $\mathrm{MCn}$ $R_{2}=600 \mathrm{~m}$.

The stability criteria are the same as in the two-mirror case:

$0 \leqslant G_{1} G_{2} \leqslant 1$.

$d_{1}, d_{2}$ and $R_{2}$ have been designed to be practically equal to $600 \mathrm{~m}$. This gives

$G_{1}=-0.875$,

$G_{2}=-1$,

$d_{0}=75 \mathrm{~m}$.

The radii of curvature of the mirrors, as they have been delivered by the manufacturers are

MFn: $666 \mathrm{~m}, \mathrm{MCn}: 636 \mathrm{~m}, \mathrm{MFe}: 686.7 \mathrm{~m}$, and MCe: $622 \mathrm{~m}$.

To improve the symmetry and thus the interference quality of the interferometer, the back side of MFE is heated to bring the curvature of the front face close to $666 \mathrm{~m}$, the radius of curvature of MFn, the other far mirror. As Eq. (18) shows, this is sufficient to match $w_{0}$ for the two arms.

For the real mirrors we have

$$
\begin{aligned}
& G_{1 \text { north }}=-0.80, \quad G_{2 \text { north }}=-0.99, \quad d_{0 \text { north }}=119.0 \mathrm{~m}, \\
& G_{\text {least }}=-0.75, \quad G_{2 \text { east }}=-0.99, \quad d_{0 \text { east }}=151.5 \mathrm{~m} .
\end{aligned}
$$


The beam-size is also given by relations very similar to those for the two-mirror case; the only difference is that the mirror separation $\ell$ is replaced by $d_{0}$. The beam radius at the power recycling mirror is given by $w_{0}$ as defined by the relation:

$w_{0}^{2}=\frac{\lambda d_{0}}{\pi} \sqrt{\frac{G_{2}}{G_{1}\left(1-G_{1} G_{2}\right)}}$,

and that at the near mirror (MCe or MCn) by $w_{2}$

$w_{2}^{2}=\frac{\lambda d_{0}}{\pi} \sqrt{\frac{G_{1}}{G_{2}\left(1-G_{1} G_{2}\right)}}$.

For completeness the beam-size at MFe in the case of a flat recycling mirror is also given here:

$w_{1}=w_{0} \sqrt{1+\left(\frac{\lambda d_{1}}{\pi w_{0}^{2}}\right)^{2}}$.

For the design values as given above the numbers for the beam-radii are

$w_{0}=8.764 \times 10^{-3} \mathrm{~m}$,

$w_{1}=24.72 \times 10^{-3} \mathrm{~m}$,

$w_{2}=8.198 \times 10^{-3} \mathrm{~m}$.

The beam diameter was chosen to be small at the input by using a flat recycling mirror, thus forcing the eigenmode to have a waist there. In addition the layout is close to a stability limit which is defined by $R_{1}=\ell$, when a flat recycling mirror and $R_{2}=d_{1}=d_{2}=\ell$ have been kept fixed. The implication of the vicinity to this stability limit becomes clear for instance when defining the deviation of $R_{1}$ from the geometrical armlength $\ell$ by $\Delta_{1}$ :

$R_{1}=\ell+\Delta_{1}$.

Then we get

$d_{0} \approx 2 \Delta_{1}$,

and, after some algebra

$w_{0} \approx \frac{\lambda}{\pi} \sqrt{2 \Delta_{1} \ell}$.

For $R_{1} \rightarrow \ell$ the beam radius at the recycling mirror vanishes, whereas the beam diameter at the far mirror grows correspondingly.

As far as beam-size and Gouy phase in GEO 600 is concerned, the deviation of $R_{2}$ from the design value is of less importance than that of $R_{1}$. This becomes clear from the $G$ factors

$G_{1} \approx-1+2 \Delta_{1}\left(\frac{1}{\ell}-\frac{1}{R_{0}}\right)$,
$G_{2} \approx-1+2 \Delta_{1}\left(\frac{1}{\ell}-\frac{1}{R_{2}}\right)$.

For a flat recycling mirror $\left(R_{0}=\infty\right)$, we have

$G_{1} \approx-1+2 \Delta_{1} / \ell$, independent from $R_{2}$. The design value for $R_{2}$ in GEO 600 is $R_{2}=\ell=600 \mathrm{~m}$, and therefore

$G_{2} \approx-1$.

The product $G_{1} G_{2}$, defining the stability of the recycling cavities, is thus mostly governed by $\Delta_{1}$

$G_{1} G_{2} \approx 1-2 \Delta_{1} / \ell$.

This shows the narrow tolerance for $R_{1}$; especially it is not allowed to approach the armlength $\ell$. For $R_{2}$ the conditions are less tight. The recycling cavities formed by the components as delivered by the manufacturers are therefore well within the stability limits.

\section{Surface figure of the core optics}

In addition to the tolerances for the radii of curvature of the component surfaces there are the requirements for the so called surface figure - the acceptable deviation from the desired surface shape, which in most cases is given by an ideal sphere or an ideal flat.

These tolerances are defined by the requirements of

1. good interference quality,

2. low scattering, and

3. minimum coupling to higher-order modes.

To describe the surface figure quantitatively, it would be best to look at the spectrum of the surface deformations as a function of the spatial wavelength of the deformation. A comprehensive description of the influence of wavefront irregularities on the performance of the interferometer is fairly complicated, but some simple statements are possible.

Surface irregularities with spatial wavelengths $\Lambda_{\mathrm{s}}$ smaller than $\lambda \ell / w_{\mathrm{m}}(\lambda=$ optical wavelength, $\ell=$ interferometer armlength, and $w_{\mathrm{m}}=$ radius of the mirror substrate) causes the light to be scattered such as to miss the mirror to be hit next by the light. This amount of light is totally lost for the interferometer. The corresponding cutoff wavelength $\Lambda_{\mathrm{s}}$ for GEO 600 is $6 \mathrm{~mm}$. Surface deformations with spatial wavelengths longer than $\Lambda_{\mathrm{s}}$ deform the wavefront in a way as to couple light into higher-order modes of the recycling cavities. Depending on the geometry and finesse of the recycling cavities, higher-order modes are suppressed to a large extent.

Unfortunately there are no spectra of the surfaces of the GEO core optics, but instead some interferograms to show the quality of the surfaces before coating. The specifications for the polisher (General Optics), typically have been: Flat or spherical, respectively, better than $5 \mathrm{~nm} \mathrm{rms}$ for the central area of the component $(220 \mathrm{~mm}$ diameter for the beam-splitter and $150 \mathrm{~mm}$ diameter for the mirrors), and better than $1 \AA$ microroughness in that area. The microroughness gives the rms deviation from a smooth reference as measured over an area of $\mathrm{mm}$ scale. These specifications have been well met by the manufacturer. The two quoted 
numbers allow an estimate of the quality of the surface shape, as the linear spectrum of the surface deformation of such high quality components shows in most cases a dependence roughly proportional to $\Lambda$ (see the LIGO pathfinder report [11], and results obtained with mirrors polished for us by ZEISS [12]). The amount of light missing the cavity mirrors because of surface scattering can thus be estimated to be in the order of a few $10^{-5}$.

The GEO mirrors have been coated by REO (the American company Research Electro Optics). After coating no measurement of the surface figure has been performed. Measurements of previously polished and well characterised substrates, including some made by Zeiss in the early 1990s, and those made for LIGO in the LIGO pathfinder program, were carried out. Repeat mesurements after coating showed that, except on a scale comparable to the mirror diameter, there was little extra deformation due to the coating process.

\section{Tolerance for misorientation of far mirror}

The far mirrors are the only components $600 \mathrm{~m}$ away from the central tank. The other critical components of the main optics all sit together inside the central building with a common concrete floor, giving rise to some common mode rejection of mechanical disturbances. In addition, for nearby components the control tasks are easier. Insensitivity to fluctuations in orientation of the far mirrors is therefore highly desirable. This insensitivity was realized by choosing the radius of curvature of the near mirrors equal to the geometrical armlength $\ell$ - in our case $600 \mathrm{~m}$. The far mirror sits now in the centre of curvature of the near mirror surface; therefore the beam hits the near mirror perpendicularly, independent of the orientation of the far mirror. The beam is reflected back into itself as desired. Even though the delivered mirrors deviated from the ideal $600 \mathrm{~m}$ curvature, the tolerable misorientation of the far mirrors is set by the demand not to spill light off the near mirrors, rather than by the interference quality. The auto-alignment of the interferometer keeps the angular misalignment of the far mirrors well below the thus tolerable value of almost $10^{-4} \mathrm{rad}$.

\section{Capability for high power load}

The shot noise limit for the sensitivity of the interferometer improves proportional to the square-root of the light power sensing the mirror separation, provided the increasing power does not cause too much degradation of the fringe contrast. Therefore the setup was designed to stand as high a light power as possible. The present light power level in GEO 600 is about $3 \mathrm{~kW}$, corresponding to the particular experimental situation. Without major changes it can be increased to somewhere between 7.5 and $10 \mathrm{~kW}$. For the planned upgrade of GEO 600, the so called GEO-HF, a 10 times higher light power is envisaged [13].
Fortunately even at those high light powers the noise due to fluctuating light pressure onto the mirrors is negligible.

There are mainly two thermal effects to distort the wavefront of the laser beam inside the interferometer - thermal expansion of the substrates locally heated by the laser beam, and thermal lensing when the light is transmitted through a substrate where absorption of light locally heats the material and thus changes the index of refraction [14]. The relevant quantities for fused silica, the material of the GEO-mirrors and beam-splitters, are $\alpha / \kappa=0.33 \times$ $10^{-6}(\mathrm{~m} / \mathrm{W})$ and $\beta / \kappa=10^{-5}(\mathrm{~m} / \mathrm{W})$. Here, $\alpha$ is the thermal expansion coefficient, $\beta$ the temperature dependence of the index of refraction, and $\kappa$ the thermal conductivity. The thermal lensing effect is therefore 33 times more effective than the thermal expansion.

Birefringence was not yet observed in GEO 600. Thermally induced birefringence can be estimated to be of much less importance in GEO 600 than thermally induced wavefront-distortions [15].

\subsection{Absorption inside the beam-splitter}

The most critical component in GEO 600 with respect to thermal effects is the beam-splitter, as a high light power is transmitted through the substrate. As we will see below, approximately $5 \mathrm{ppm}$ of the transmitted light is absorbed, and the highly reflective coatings of the mirrors typically absorb about 2 ppm [16]. As it turns out, wavefront deformation via thermal lensing in the beam-splitter dominates by far the thermal expansion effect of the mirror surfaces inside the recycling cavities.

Absorption of light with $1064 \mathrm{~nm}$ wavelength in very pure fused silica is dominated by absorption at $\mathrm{OH}$-groups [17]. The beam-splitter in GEO 600 is therefore made from Suprasil 311 SV, a particular grade of fused silica with low $\mathrm{OH}$-content. It was manufactured by the German company Heraeus, according to an initiative of scientists from the French-Italian detector VIRGO. The absorption quoted by the manufacturer was $<0.5 \mathrm{ppm} / \mathrm{cm}$. This measurement of the absorption [18] was limited by the noise in the setup used, and the real absorption was not known. Still, this was the lowest value reported so far. The effect of beam-splitter absorption affects the stability of the recycling cavities and the technique employed to deduce the absorption in situ is briefly described here.

In GEO 600, for this particular experiment the lightpower running from the power recycling mirror towards the beam-splitter was $1.5 \mathrm{~kW}$, and thermal lensing inside the beam-splitter started to change the shape of the eigenmodes of the recycling cavities noticeably. This allowed us to determine the absorption to be $0.25 \mathrm{ppm} / \mathrm{cm}$, corresponding to an absorbed light power of $3.4 \mathrm{~mW}$. The procedure is described in another paper [19] and is summarized below.

For fused silica the thermally induced lens inside the substrate is a convex one. As one can see from Fig. 1, only light entering the east arm has to go through the beam-splitter; 
the north arm gets its light from reflection at the beam-splitter. A cavity formed by the power recycling mirror and the east arm therefore contains the thermally induced lens inside the beam-splitter, whereas the cavity made from the power recycling mirror and the north arm does not. The interference pattern at the output therefore changes with increasing thermal lens. For a quantitative description, the simulation program FINESSE [20] has been used to reconstruct the shape of the output beam as a function of the strength of the thermal lens. The observed output beam was obtained for an assumed absorption of $0.25 \mathrm{ppm} / \mathrm{cm}$. This is the lowest value reported so far in the literature for light absorption inside fused silica.

\subsection{Light power limits for the present setup}

As stated above, the dominant thermal effect inside the recycling cavities is the thermal lens inside the beam-splitter. This thermal lens is equivalent to a concave power recycling mirror MPR, as far as the properties of the power recycling cavity are concerned; in other words: the lens can be replaced by the introduction of a finite $R_{0}$ for MPR. Increasing light power makes the absolute value of $R_{0}$ smaller; $w_{0}$ and $w_{2}$, the beam-radii at MPR and MCe, respectively, would grow, whereas the beam diameter $2 w_{1}$ at the far mirror would decrease. A stability limit is defined by $R_{0} \approx \ell=600 \mathrm{~m}$. Approaching this limit eventually makes the beam diameter at all three involved mirrors grow including $w_{1}$ at the far mirror. A practical limit for the present geometry is set for instance by the hole in the reaction mass of the power recycling mirror, through which the beam has to pass. If we allow a few ppm to be cut off, the beam radius $w_{0}$ is limited to about $12 \mathrm{~mm}$. The corresponding other numbers are: $R_{0}=810 \mathrm{~m}$ and a sagitta of the wavefront of $1.3 \times 10^{-7} \mathrm{~m}$. This last value is reached for a light power of $46 \mathrm{~kW}$ inside the power recycling cavity, or $23 \mathrm{~kW}$ in each arm. In order to achieve good interference between the beams of the two arms, the beam radius $w_{0}$ of the power recycling cavity formed with the north arm also needs to be increased to $12 \mathrm{~mm}$, to match that obtained with the east arm. This could be done by increasing the radius of curvature of the far mirror in the north arm from $640 \mathrm{~m}$ to $700 \mathrm{~m}$, for instance by using MFe, the far mirror in the east arm, as far mirror MFn in the north arm.

One might think of reducing the beam diameter at MPR as defined by the eigenmode in the east arm, by means of reducing the radius of curvature of the east far mirror close to the limiting $600 \mathrm{~m}$. This would work as far as the beam diameters are concerned. But the Gouy phase (see below) would come close to the forbidden value of $\pi$, permitting higher modes to be resonant simultaneously with the fundamental mode.

The power- and signal recycling cavities in GEO 600 are to a large extent stable with respect to deviations from the design values of the radii of curvature. An example: For the design values, the higher modes of order 9 are close to resonance. The strongly deviating real values, as realized by the manufacturers, bring the higher modes of order 6 close to resonance. In case this causes stability problems, the thermally induced change in radius of curvature of a critical component, in most cases the far mirror, may help [7]. The stability also helps the toleration of a certain amount of thermal effects, as for instance a change in radius of curvature of the mirrors or an introduction of a thermal lens in the beam-splitter due to local heating by the illuminating laser beam.

The essential quantity to describe the stability is the so called Gouy phase. In addition to a plane wave case, for the fundamental mode of laser light there is an additional phase, the Gouy phase $\psi$, when going from MPR via $\mathrm{MFe}$ to MCe. It is given by

$\psi=\arccos \left(-\sqrt{G_{1} G_{2}}\right)$.

For a total round-trip $L=4 \ell$ (MPR to MPR) one has to add $\Theta=2 \psi$ to get the proper phase of light in the eigenmode. The frequency $f(0)$ of the fundamental mode, the number of wavelengths $n_{0}$ in a roundtrip and the Gouy phase $\Theta$ are related by

$f(0)=\frac{c}{L}\left(n_{0}+\frac{\Theta}{2 \pi}\right)$.

Exactly as in the case of a two-mirror cavity [8], modes of order $n$ differ in Gouy phase relative to the fundamental mode by $n \psi$ or $n \Theta$, respectively. In order to avoid resonance of modes of order $n, n \Theta /(2 \pi)$ needs to differ from an integer; the deviation should be equivalent to a few times the optical bandwidth of the resonance. The frequency shift to move from the fundamental mode resonance to resonance at $(m, n)$ mode is given by

$f(n, m)-f(0,0)=(n+m) \frac{c}{2 \pi L} \Theta$,

with $L=4 \ell$, and $\ell=$ geometrical armlength of $600 \mathrm{~m}$. For the GEO 600 design values one gets $\Theta /(2 \pi)=0.8850$. The values obtained with the real components and their fairly strong deviation from design values (see above) are: $\Theta_{\text {north }} /(2 \pi)=0.85, \Theta_{\text {east }} /(2 \pi)=0.83$. The values denoted by "east" or "north" indicate which arm was used to obtain this number.

The power recycling cavity as shown in Fig. 1 has a finesse of $F=2350$. The free spectral range FSR $=c / L$ is $125 \mathrm{kHz}$. This gives an optical bandwidth FWHM $=\mathrm{FSR} / F=53 \mathrm{~Hz}$. A higher mode of order $n$ is therefore close to resonance when $n \Theta /(2 \pi)$ comes into the vicinity of an integer within $10^{-3}$. The lowest higher-order mode coming close to this condition is that of order 6 . In case this causes problems, a slight variation of $R_{1}$ (see Eqs. (23) and (24)) can help. Thus, all relevant higher modes can be prevented from being resonant.

\section{The mode-cleaners}

In addition to the main interferometer there are the so called mode-cleaners, which may be considered as being 


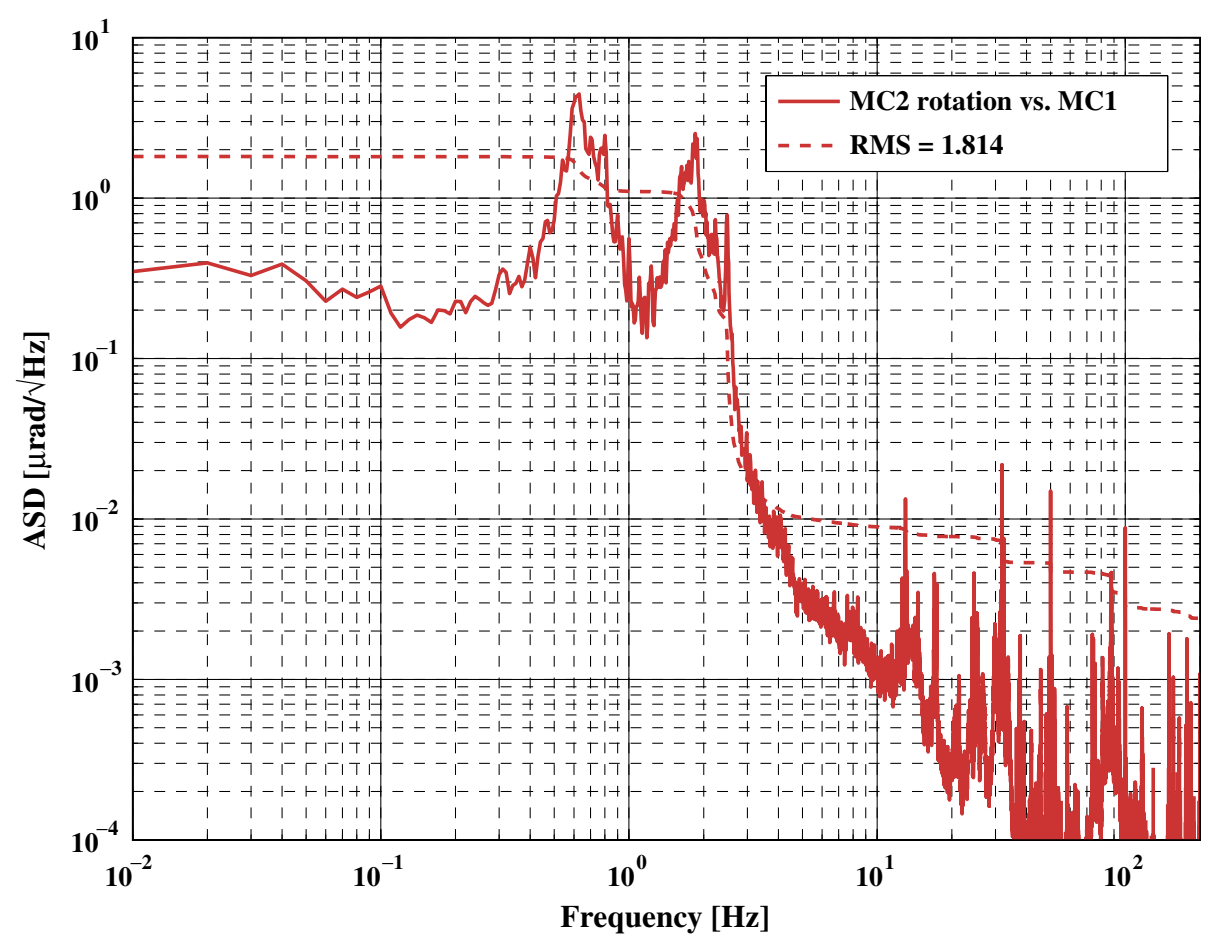

Fig. 3. Fluctuations in beam orientation in front of the mode-cleaners.

part of the core optics. They are inserted between the laser and the interferometer, in order to suppress spurious signals caused by geometry fluctuations of the laser beam in combination with imperfections of the interferometer [8].

The main geometry fluctuations of the laser beam are those in position and in orientation. A lateral displacement can be described by the addition of a spatial 01 mode in phase with the fundamental mode. An angular displacement is equivalent to the addition of such a mode with $\pi / 2$ phase difference relative to the fundamental mode. Such fluctuations lead to spurious interferometer signals in combination with imperfections of the interferometer. An example: A lateral displacement $\delta x$ of the beam in combination with an angular displacement $\delta \alpha$ of the beamsplitter (with respect to the symmetry plane between the two interferometer arms) gives rise to an armlength-difference of $4 \delta x \delta \alpha$.

Thus a fluctuating input beam position leads to a false displacement signal. In order to reduce these spurious signals, it has become common use to send the laser beam through a so called mode-selector. This mode-selector transmits the fundamental mode, and reduces higher-order modes by a factor $1 / S=\pi / F$, with $F=$ finesse of the modeselector. Geometrical beam fluctuations are then reduced by the same factor.

In order to understand the requirements in GEO 600, in Fig. 3 a typical spectrum of the angular displacements of the GEO laser beam is shown. The dynamic angular displacement of the beam-splitter has been determined to be in the order of $10^{-7} \mathrm{rad}$. Injecting at $120 \mathrm{~Hz}$ an artificial beam jitter with an amplitude of $1 \mathrm{nrad}$ has been measured to produce in the GEO 600 detector an $h$ signal of $6 \times 10^{-20}$.

In order to reduce the interferometer signals inferred by spurious beam jitter below the envisaged sensitivity level of $h=8 \times 10^{-23} / \sqrt{\mathrm{Hz}}$, a suppression of the beam fluctuations by at least a factor of $10^{4}$ has to be accomplished.

The particular mode-cleaners used for this purpose in GEO 600 have already been described in earlier papers $[21,22]$. The mirrors are made from Suprasil 1, $100 \mathrm{~mm}$ diameter and $50 \mathrm{~mm}$ thick. The two mirrors sitting close to each other are flat, the other one has a radius of curvature of $6.72 \mathrm{~m}$. The round-trip path-length for the first mode-cleaner was chosen to be $8 \mathrm{~m}$, for the second one $8.1 \mathrm{~m}$. The different lengths allowed us to sufficiently separate the eigenfrequencies from multiples of the free spectral ranges of the cavities, which is the requirement for effective spatial filtering by the sequential mode-cleaners.

For practical reasons the finesse of a mode-cleaner is limited to values below $10^{4}$. It was therefore necessary to implement two mode-cleaners in series - the suppression factors are then multiplied. The finesses of the two mode-cleaners, 2700 and 1900 , respectively, were due to the coating process as applied by the manufacturer. Together they show a suppression factor of $5.2 \times 10^{5}$ - leaving some room for reducing the relatively high finesse values of the mode-cleaners.

\section{Summary}

The optical design of the GEO 600 interferometer has achieved the planned goals of reaching high light power, providing stable operation, and demonstrating the effective 
combination of power- and signal recycling. For the envisaged upgrade of GEO 600 to GEO-HF, the present lightpower of $3 \mathrm{~kW}$ will be increased by up to an order of magnitude. This is expected to be possible when some compensation of the thermal lensing effect inside the beamsplitter is done by means of thermal correction of the wavefront.

\section{Acknowledgments}

The authors gratefully acknowledge the support of The Particle Physics and Astronomy Research Council of the United Kingdom, the Max-Planck Society and the State of Niedersachsen (Germany).

\section{References}

[1] H. Grote, A. Freise, M. Malec, G. Heinzel, B. Willke, H. Lück, K.A. Strain, J. Hough, K. Danzmann, Class. Quantum Grav. 21 (2004) 473.

[2] H. Lück et al., Class. Quantum Grav. 23 (2006) S71.

[3] D. Sigg et al., Class. Quantum Grav. 21 (2004) 409.

[4] F. Acernese et al., Class. Quantum Grav. 21 (2004) 385.

[5] R. TakahashiThe TAMA Collaboration, Class. Quantum Grav. 21 (2004) 403.

[6] S. Hild, Presentation given on the Gravitational Wave Advanced Detector Workshop, Elba 2006, Available from: <http:// www.ligo.caltech.edu/docs/G/G060342-00/G060342-00.pdf>.

[7] H. Lück, A. Freise, S. Goßler, S. Hild, K. Kawabe, K. Danzmann, Class. Quantum Grav. 19 (2002) 1803.
[8] A. Rüdiger, R. Schilling, L. Schnupp, W. Winkler, H. Billing, K. Maischberger, Opt. Acta 28 (1981) 641.

[9] H. Kogelnik, Bell Syst. Tech. J. (March) (1965) 455.

[10] A.E. Siegmann, LASERS, University Science Books, Mill Valley, 1986.

[11] David I. Farrant, Achim J. Leistner, Bozenko F. Oreb, Mark A. Suchting, Christopher J. Walsh, in: H. Philip Stahl (Ed.), Proc. SPIE, vol. 3134, Optical Manufacturing and Testing II (SPIE Homepage), October 1997, p. 79.

[12] Unpublished.

[13] B. Willke et al., in: Amaldi Conference, 2005.

[14] W. Winkler, K. Danzmann, A. Rüdiger, R. Schilling, Phys. Rev. A 44 (11) (1991) 7022.

[15] W. Winkler, A. Rüdiger, R. Schilling, K.A. Strain, K. Danzmann, Opt. Commun. 112 (1994) 245.

[16] J.M. Mackowski, SMA Lyon, Private Communication.

[17] Standard Optics Information, Heraeus, POL-O/433M.

[18] V. Loriette, C. Boccara, Appl. Opt. 42 (2003) 649.

[19] S. Hild, H. Lück, W. Winkler, K. Strain, H. Grote, J. Smith, M. Malec, M. Hewitson, B. Willke, J. Hough, K. Danzmann, Appl. Opt. 45 (2006) 7269.

[20] A. Freise et al., Class. Quantum Grav. 21 (2004) 1067.

[21] S. Gossler, M.M. Casey, A. Freise, H. Grote, H. Lck, P. McNamara, M.V. Plissi, D.I. Robertson, N.A. Robertson, K. Skeldon, K.A. Strain, C.I. Torrie, H. Ward, B. Willke, J. Hough, K. Danzmann, Class. Quantum Grav. 19 (2002) 1835.

[22] S. Gossler, M.M. Casey, A. Freise, A. Grant, H. Grote, G. Heinzel, M. Heurs, M.E. Husman, K. Ktter, V. Leonhardt, H. Lck, M. Malec, K. Mossavi, S. Nagano, P.W. McNamara, M.V. Plissi, V. Quetschke, D.I. Robertson, N.A. Robertson, A. Rüdiger, R. Schilling, K.D. Skeldon, K.A. Strain, C.I. Torrie, H. Ward, U. Weiland, B. Willke, W. Winkler, J. Hough, K. Danzmann, Rev. Sci. Instrum. 74 (2003) 3787. 